\title{
Balkanologie
}

Balkanologie Revue d'études pluridisciplinaires

Vol. III, n² | 1999

Volume III Numéro 2

\section{Formation nationale et nationalisme dans l'aire de peuplement albanais}

Albert Doja

\section{(2) OpenEdition}

12 Journals

Édition électronique

URL : http://journals.openedition.org/balkanologie/738

DOI : 10.4000/balkanologie.738

ISSN : 1965-0582

Éditeur

Association française d'études sur les Balkans (Afebalk)

Édition imprimée

Date de publication : 1 décembre 1999

ISSN : 1279-7952

Référence électronique

Albert Doja, « Formation nationale et nationalisme dans l'aire de peuplement albanais », Balkanologie [En ligne], Vol. III, n² I 1999, mis en ligne le 22 juin 2010, consulté le 17 décembre 2020. URL : http:// journals.openedition.org/balkanologie/738; DOI : https://doi.org/10.4000/balkanologie.738

Ce document a été généré automatiquement le 17 décembre 2020.

(c) Tous droits réservés 


\title{
Formation nationale et nationalisme dans l'aire de peuplement albanais
}

\author{
Albert Doja
}

Les événements dramatiques qui ont secoué l'Europe durant dix années à la suite du démembrement de la Yougoslavie, surtout le dernier épisode retentissant qui a fait s'affronter les Serbes et les Albanais pour la paternité du Kosovo, ont impliqué aussi l'ensemble de la communauté internationale pour la défense d'un certain modèle de société et de relations entre groupes ethniques. Les opinions publiques en revanche, abasourdies par les bruits médiatiques et intellectualistes, n'ont toujours pas saisi la signification et les raisons du conflit, que tout le monde espère voir finir une fois pour toutes avec ce dernier et final épisode sanglant. Après une introduction sur les caractéristiques de la région définie comme une aire culturelle, je passerai en revue l'histoire du mouvement national et la formation de la nation albanaise au tournant du siècle, en essayant de rendre compte de l'affrontement des nationalismes serbe et albanais qui n'ont pas manqué de s'exacerber à tout moment. En intégrant l'approche anthropologique aux considérations historiques et géopolitiques sur la région et la culture albanaise, l'article tentera de poser une question qui me paraît essentielle pour la compréhension des phénomènes actuels, à savoir: l'héritage historique et les identités culturelles peuvent-ils raisonnablement, sinon justifier, au moins expliquer les conflits ethniques et le nationalisme, ou au contraire servent-ils tout simplement à déterminer et au mieux à rationaliser les relations interethniques entre groupes sociaux?

Contrairement aux suppositions anciennes, les groupes ethniques et les cultures n'ont jamais été des entités d'une durée temporelle constante ni d'une auto-définition dérivée indépendamment des contacts extérieurs. Ils se sont formés et articulés au contact les uns des autres, parfois en conflit, parfois pacifiquement à travers les divisions de travail. Les cultures tribales, décrites comme apparemment insulaires par les anciens anthropologues, étaient rarement aussi isolées et elles le sont considérablement moins aujourd'hui. Le vieux concept de tribu est largement remplacé par le terme "groupe ethnique", utilisé souvent comme synonyme de "culture". Le 
changement décisif n'est donc pas la disparition des groupes ethniques mais le degré plus élevé de l'interaction entre eux-mêmes. Un résultat remarquable, qui semble échapper aux théories classiques sur la modernisation, est le renforcement des solidarités locales et particularistes. Les nationalistes et les apologistes des cultures locales donnent toujours la proéminence à la durée temporelle constante (l'inaltérable essence des origines) des entités ethniques dont ils font la propagande. En revanche, l'anthropologie actuelle s'appuie sur les groupes ethniques et les cultures en tant que "constructions". Elles sont sujettes à de multiples pressions adaptatives, elles se transforment en conséquence et peuvent devenir sujettes à des manipulations délibérées.

\section{L'aire culturelle}

3 La considération de la région comme une aire culturelle se définit comme un champ composite. L'aire de peuplement albanais n'est pas seulement celle d'un pays de l'Europe de l'est des politologues contemporains, ni celle d'un pays de l'Europe balkanique post-byzantine ou ex-ottomane, pas plus le lieu d'expansion de certains modèles occidentaux ou d'enracinement d'identités locales, mais bien tout cela à la fois. Dans le contexte européen, les sociétés du sud-est, à l'intersection du monde méditerranéen et du reste de l'Europe, reflètent comme un miroir grossissant la civilisation "moderne" dans son ensemble. Dans une perspective comparative, le domaine géopolitique de l'Europe du sud-est peut faire l'objet d'investigations approfondies, dont l'explication ne repose que sur la définition de l'interdépendance de divers facteurs aboutissant parfois à des contradictions historiques. Au long de l'évolution historique, les facteurs politiques, culturels et idéologiques (tradition grécoromaine et byzantine, influences orientales, tendances d'occidentalisation, etc.) ont toujours joué un rôle plus important que les conditions sociales et économiques, contrairement à ce qu'il en fut en Europe occidentale. Dans ce sens, ces sociétés nous invitent à une révision de la manière dont l'ensemble de la civilisation européenne se conçoit.

4 L'histoire culturelle, sociale et géopolitique des sociétés sud-est européennes et de la société albanaise en particulier constitue l'un des développements de ce qu'on a appelé la "question d'Orient". À moins qu'on ne la situe dans une perspective plus longue ${ }^{1}$, on définit couramment celle-ci comme l'ensemble des problèmes géopolitiques posés, depuis le milieu du XVIII ${ }^{\text {ème }}$ siècle, par la décomposition de l'Empire ottoman. La forme même de l'expression "question d'Orient" indique bien son origine. Formulée par des hommes d'États, diplomates et intellectuels occidentaux, elle dénote un impérialisme ethnocentrique dans lequel les grandes puissances, tour à tour alliées et rivales, poursuivent à la fois le maintien d'un équilibre européen et la réalisation de leurs objectifs propres. Dans cette optique, la question d'Orient se réduisait à une série de problèmes politiques à régler. On peut cependant, en changeant de point de vue, donner à cette expression une signification plus générale, celle d'une crise interne à une "région intermédiaire"2, située entre l'Occident, l'Orient et l'Afrique, et centrée sur la Méditerranée. Prendre conscience de son unité dynamique, c'est effacer la coupure entre l'est et l'ouest, le sud et le nord et reconnaître le caractère synthétique de la civilisation européenne, assimilant les traditions romaine et hellénique, byzantine et ottomane, slave et germanique, chrétienne et islamique, démocratique et autocratique. 
5 Depuis des époques lointaines, des mouvements de populations brutaux ou pacifiques ont perduré, dont l'histoire a dressé une liste impressionnante. Ces vagues successives se sont mêlées en une alchimie d'intégrations et de rejets qui a fait naître les peuples sud-est européens actuels. Le modèle républicain à la française a voulu les enfermer dans des États-nations mais ils débordent tous de leurs frontières trop rigides. Porteurs d'une culture millénaire ou pluri-millénaire, ils forment dans la péninsule le tissu humain de base sur lequel sont venus se greffer les nouveaux apports des conquérants ou des colons de l'époque historique.

6 L'un des principaux substrats du peuplement sud-est européen est constitué par des tribus illyro-thraces d'origine indo-européenne. Alors que les Grecs ont fait mouvement vers le sud au milieu du II ${ }^{\text {ème }}$ millénaire avant J.-C., en se mélangeant aux autochtones policés par la Crète pour donner naissance à la civilisation mycénienne et par la suite à celle de l'Athènes classique, les Illyriens se sont perpétué durant deux millénaires non sans de nombreux apports successifs, en particulier celui des Latins et plus tard celui des Slaves, pour donner naissance au peuple albanais. La colonisation romaine s'est développée à partir de la Grèce et si la civilisation gréco-romaine préservait la langue et la culture d'époque hellénistique, les autres populations intégrées à l'Empire, à l'exception des Albanais, ont été romanisées. La pénétration des Slaves entraîna une slavisation progressive de toutes les populations sud-est européennes, à laquelle ont échappé à nouveau les Albanais, mais aussi les Grecs et les Roumains, qui ont assimilé les nouveaux venus. Byzance succède à Rome comme État impérial et domine la région jusqu'au $\mathrm{XV}^{\mathrm{ème}}$ siècle. Durant toute une période de dissidences, surtout après le schisme cérulaire en 1054, les Églises orthodoxes d'Orient partagent la région avec l'Église catholique de Rome. Avec la chute de l'Empire byzantin, c'est le tour de l'Empire ottoman auquel se rattache une tradition islamique. Le christianisme a subsisté, mais contrôlé et persécuté par les Ottomans, disputés parfois par l'Empire habsbourgeois, qui renouait avec la tradition catholique.

7 De ce formidable brassage de populations subsiste une très grande diversité culturelle, ethnique, linguistique, religieuse et politique. C'est le lieu de partage par excellence entre les Empires romains d'Orient et d'Occident, entre l'islam et le christianisme, entre l'orthodoxie et le catholicisme, entre les blocs du Traité de l'Atlantique Nord et du Pacte de Varsovie. Au cœur même de ce foyer se trouve, sûrement plus que tout autre composante, le cas albanais "faible chaînon" (Braudel) entre le monde latin, grec et slave. Car le seul exemple albanais renferme en soi probablement l'essentiel d'une série de problématiques comparatives culturelles, historiques et géopolitiques. L'approche d'une population partagée de part et d'autre d'une frontière politique (les Albanais et les Kosovars), recoupant structures sociales et politiques, nationalismes et religions, relations interethniques et migrations, est certainement très riche d'enseignements.

8 Il se révèle pourtant quasiment impossible d'articuler ensemble les données localisées dans le sud-est européen, malgré les tentatives, notamment des ethnologues, de mettre en ordre la diversité sud-est européenne : elles ont été effectuées selon des critères hétérogènes entre eux. Les quelques découpages empiriques proposés par les anthropologues à l'échelle européenne ${ }^{3}$ ne se superposent que grossièrement aux cartes de l'Europe du sud-est, susceptibles d'être dressées en fonction de critères géographiques, de la répartition des éléments de culture matérielle, des familles linguistiques, des aires religieuses ou encore des ensembles historiques, aux frontières 
floues et changeantes, tels qu'ils portent les marques accumulées des événements majeurs du passé sud-est européen.

Ces découpages ne coïncident pas davantage, même s'ils y font souvent référence, avec la carte des substrats "ethniques", qui renvoie davantage aux illusions de la "chasse aux ancêtres" qu'à une réalité pertinente. La diversité balkanique est effectivement renforcée par le fait que les populations données pour homogènes par la tradition des études historiques, voire ethnologiques, ont pour cadres d'identité collective des sousensembles sociaux et territoriaux. Il serait trop simple d'imaginer, à la manière des historiens nationalistes du XIX ${ }^{\text {ème }}$ siècle, une stabilité de blocs ethniques justifiant des frontières modernes intangibles. D'ailleurs, le lien ethnique n'est pas nécessairement déterminant pour rendre compte de la particularité des genres de vie. Ainsi on pourrait multiplier les exemples qui mettent en évidence le poids de l'histoire dans les formations des entités culturelles d'aujourd'hui. La conquête ottomane, avant tout, a ébranlé les groupes ethniques. La politique des sultans n'a pas reculé devant des transferts de populations et des entreprises de colonisation, tandis que l'activité économique dans les frontières d'un empire étendu à trois continents se traduisait par d'amples mouvements de populations. C'est surtout à partir du XIX ${ }^{\text {ème }}$ siècle que l'appartenance ethnique est mise en avant dans le sud-est européen pour soutenir les prétentions à l'indépendance. Les revendications s'expriment alors dans la littérature, dans les recherches et les publications folkloriques ou l'organisation des musées ethnographiques.

Il faudrait ainsi, paradoxalement peut-être, rechercher une insaisissable unité, et donc la pertinence de l'idée d'une culture, d'une civilisation et d'une histoire albanaise et sud-est européenne, dans cette diversité même, en quelque sorte dans une coexistence des dissemblances, préservées plus fortement qu'ailleurs. La démarche, ainsi définie, pourrait conférer à cette partie de l'Europe un visage distinct tout en déterminant une problématique qui lui soit proprement applicable. Les études sud-est européennes sont extrêmement vastes et complexes. Sujette aux influences extérieures rivales autant qu'aux pressions internes, l'aire sud-est européenne est un champ d'expérience pour des systèmes alternatifs.

11 La civilisation sud-est européenne devrait être comprise dans son ensemble, chacune de ses composantes ayant fait partie, à une époque historique donnée, d'une construction culturelle, religieuse ou politique plus vaste. Elle se situe à une aire de confrontation sur laquelle des Empires et des États aspirant à la puissance ont constamment débordé, où orthodoxie, catholicisme et islam se touchent et s'entremêlent, par-dessus des découpages et des espaces de coexistence ethno-culturels et ethno-linguistiques différents. Autant de particularismes et de clivages discrets, souvent ignorés ou dénaturés par le modernisme actuellement dominant dans les cultures nationales, tout comme autrefois par la volonté de nivellement des grands corps impériaux. Aujourd'hui l'anthropologie sociale et historique, la sociologie, les recherches sur les structures familiales, sur les économies traditionnelles, sur les littératures orales, ou sur les formes nombreuses de solidarité sociale et culturelle, tentent d'une manière ou d'une autre de redécouvrir et de réévaluer les différences et les ressemblances ${ }^{4}$. 


\section{Le mouvement national}

12 La formation de la nation albanaise, comme celle des autres nations balkaniques, s'est effectuée dans le cadre du démantèlement de l'Empire ottoman qui est ébranlé de l'intérieur par les mouvements nationaux tout en perdant du terrain sous les coups de la Russie et de l'Autriche-Hongrie. L'expansionnisme russe, dans sa poussée vers le sud, a débouché en 1774 sur la mer Noire, auparavant Mare Ottomanicum. Dès lors, soucieux de contrôler les détroits, il s'appuie sur les peuples balkaniques, qui recherchent également son soutien. Les puissances occidentales s'efforcent de le mettre en échec, soit en lui disputant la faveur desdits peuples, soit en s'alliant militairement avec la Porte, comme dans la guerre de Crimée (1854-1856). Contradictoirement, elles s'appliquent, soit à prolonger la survie de l'Empire ottoman, soit à encourager les mouvements nationaux qui s'y développent. Il ne s'agit pas d'ailleurs de simples mouvements d'émancipation nationale. Chez les bourgeoisies serbe, grecque, bulgare et autres, le désir de libérer la nation et d'accéder au pouvoir politique se prolonge dans un expansionnisme territorial quelque peu mégalomane, bardé de justifications historiques. Les Grecs veulent reconstituer l'Empire byzantin au nom de la Megale Idea, la "Grande Idée", les Serbes se réclament de l'empire de Dušan, les Bulgares de celui de Siméon ou de Kaloyan. Les Albanais, seuls dépourvus de références impériales, n'en revendiqueront pas moins un assez large espace, inspirés plutôt par des références ethno-linguistiques.

Dans le courant du XIX ${ }^{\text {ème }}$ siècle, quatre États nationaux, autonomes puis indépendants, se sont formés dans la partie européenne de l'Empire ottoman : la Grèce, la Serbie, le Monténégro et la Bulgarie. Après le Congrès de Berlin de 1878, l'Empire ottoman ne conserve en Europe continentale que la Thrace, la Macédoine, l'Épire, le territoire de l'Albanie actuelle, le Kosovo et le Sandjak de Novi Pazar. Ce sont pour les États balkaniques des territoires à prendre. Apparaît alors une zone d'interférences entre prétentions territoriales dont la complexité a placé la question d'Orient dans des conditions de virtualités nationales et étatiques multiples, d'incertitude totale.

Cette complexité et cette incertitude tiennent d'une part à ce que ces territoires sont les derniers à conquérir, d'autre part à ce que le processus d'affirmation nationale y est moins avancé qu'ailleurs. Les États balkaniques, produits de nationalismes asynchrones, constitués par accrétions territoriales successives, y sont en compétition pour la capture identitaire des populations, ce qui devait ouvrir la voie au contrôle politique de l'espace. Ainsi, la détermination nationale des populations chrétiennes de la Macédoine constitue un enjeu entre la Serbie, la Bulgarie et la Grèce, mais aussi entre les autorités religieuses, sans compter les ingérences extra-balkaniques ${ }^{5}$. Dans ce contexte, les Albanais sont confondus avec les Turcs s'ils sont musulmans, avec les Grecs s'ils sont orthodoxes. Quant à l'intelligentsia serbe, elle les dépeint comme de dangereux sauvages, incapables de constituer une nation et de se gouverner. Ses accointances avec la bourgeoisie française contribuent à la massive et efficace reproduction d'une description digne de la vision antique du barbare et conforme à la vision coloniale des populations d'outre-mer et du sauvage ${ }^{6}$. "Les Albanais, écrivait Marcel Mauss avec un dédain exemplaire, sont dans un stade de civilisation toujours très primitif, plus primitif certes que les Indo-Européens au moment de leur entrée dans l'histoire. $»^{7}$ 
15 Ainsi, dans la seconde moitié du XIX ${ }^{\text {ème }}$ siècle, l'affirmation nationale albanaise est cependant sinon floue du moins mal reconnue. Pour Bismarck, au Congrès de Berlin (1878), il n'y a pas de nation albanaise et l'Albanie n'est qu'une expression géographique, comme l'était l'Italie pour Metternich deux tiers de siècle plus tôt. Les sources turques ne considèrent pas le mouvement national albanais comme une réalité autonome et traitent l'histoire de ces régions comme une partie de l'histoire ottomane en général. Hors de Turquie, de nombreux historiens balkaniques considèrent aujourd'hui encore, comme les voyageurs et les diplomates d'il y a 100 ans, que la fragmentation religieuse des Albanais interdisait chez eux, au contraire des autres peuples, la formation d'une conscience nationale. Dans cette optique, le mouvement national albanais, de 1878 à 1912, ne serait que le produit d'influences et d'intérêts étrangers, ce dont on pouvait déduire que le partage du territoire d'une non-nation n'avait rien d'illégitime.

16 Il est vrai que le mouvement national albanais, à cette époque, est une réalité difficile à comprendre. Il est, avec celui des Macédoniens, le moins avancé des Balkans. En première approche, les Albanais paraissent alors ne se différencier indiscutablement de leurs voisins qu'en tant qu'albanophones, alors que la Macédoine slave n'est qu'un continuum linguistique entre Serbes et Bulgares, situation propre à alimenter entre Belgrade et Sofia un débat sans fin sur son identité ethnique. Mais la spécificité linguistique, constitutive pour les Albanais eux-mêmes de leur identité nationale, a pu être tenue par d'autres pour insignifiante, au sens propre, quand on exagérait de façon colossale les emprunts et surtout à ignorer totalement les faits de structure linguistique. C'est l'existence même d'une nation albanaise qui, pour l'Occident, paraissait difficile à saisir.

17 Les raisons de cette difficulté sont de plusieurs ordres: l'outillage conceptuel des observateurs occidentaux, leur sous-information, l'ambiance tout à fait passionnelle de cette question, enfin le comportement des Albanais eux-mêmes ${ }^{8}$. L'Occident classait alors les peuples d'Europe orientale selon une échelle de valeur qui est, en fait, une échelle de distance ethno-culturelle ressentie, dont rend compte, parmi d'autres, l'ouvrage de Marcel Mauss sur la Nation'. Le raisonnement procède également en fonction de l'utilité géopolitique supposée. C'est ainsi qu'en France on place en tête les Grecs, à cause des références à l'Antiquité classique, et les Polonais catholiques que l'on peut opposer mentalement à (ou utiliser contre) l'adversaire du moment, Russie, Prusse ou Autriche. Puis viennent les Roumains et leur néo-latinité alors en voie de consolidation à l'école de la France et de l'Italie, les Slaves orthodoxes des Balkans et notamment les Serbes, francophiles et adversaires de l'Autriche. Enfin viennent les Albanais, souvent réduits à leur majorité musulmane et mal distingués des Turcs. Le fait musulman accroît ici la distance ressentie.

Influencée par les idéologies nationales en présence, la connaissance de la structure ethnique des Balkans demeure soumise à de grandes controverses. Il en est de même du mouvement national albanais, objet des jugements les plus contradictoires. Ce mouvement national s'est longtemps cantonné dans une attitude réformatrice et défensive. D'une part, obtenir de la Porte le regroupement de tous les territoires ethniques albanais dans un unique vilayet, "province" autonome, où la langue des autochtones serait enseignée et comprise de l'administration. D'autre part, s'opposer à toute cession, par l'Empire ottoman, des terres en question. Ce qui explique en partie la difficulté, pour les observateurs étrangers, de percevoir une telle position comme 
l'expression d'un mouvement national. Les Albanais ont été poussés en avant, jusqu'à la revendication de l'indépendance complète, à la fois par les appétits territoriaux des États voisins et par l'autoritarisme des gouvernements ottomans, sourds à leur demande.

Leur mouvement national coïncide d'ailleurs avec des mouvements d'une signification toute différente: la propension traditionnelle à la révolte, mais aussi la résistance conservatrice aux tentatives de modernisation administrative décidées à Istanbul, car centralisatrices et appliquées autoritairement. Ce fut déjà le cas à l'époque des réformes connues dans l'histoire ottomane sous le nom des Tanzimat (1839-1878). Il y a dans les revendications albanaises des aspects contradictoires, qui correspondent à des clivages sociaux, idéologiques et confessionnels. Les féodaux militaires et les chefs des corporations de métier désiraient retrouver leur situation économique antérieure et leur place éminente au sommet de l'échelle sociale. Les mesures centralisatrices de la Porte étaient également perçues comme attentatoires à l'autonomie traditionnelle des Montagnes. Le reste des masses paysannes se dressait avant tout contre la charge intolérable des impôts et le lourd fardeau du nizam, le nouveau service militaire obligatoire de 9 à 11 ans, qui accéléraient leur ruine économique. Les couches citadines protestaient contre l'administration bureaucratique, contre les fonctionnaires corrompus et contre les exactions manifestes. Somme toute, il s'agissait là d'une opposition spontanée, qui ne se posait aucun objectif précis et qui n'était pas en mesure de proposer les solutions de rechange.

Toutefois, le mouvement que cette résistance engendrait n'en préparait pas moins les conditions favorables au développement du mouvement national albanais. À travers la résistance aux réformes des Tanzimat apparaissent les premiers éléments d'une conscience nationale. À la suite de ses importants succès, l'insurrection paysanne de 1847, par exemple, subit une évolution que les révoltes antérieures n'avaient pas connue. Des liens interrégionaux furent établis par les forces insurgées et un comité de coordination fut organisé par les chefs des différentes régions. De plus, l'idée de l'unité nationale commença à germer, car les insurgés déclaraient maintenant qu'ils combattaient non plus pour leurs intérêts locaux mais pour tous les vilayets d'Albanie. Ce sont précisément ces nouveaux phénomènes dans l'évolution des idées et l'établissement des liens interrégionaux qui ont laissé une masse de chants populaires à caractère historique et national dans la tradition culturelle des Albanais ${ }^{10}$.

21 Le retard et les contradictions du mouvement national albanais s'expliquent par des facteurs internes, division confessionnelle, majorité musulmane culturellement plus proche des Ottomans, retard culturel et retard du développement économique et social qui rend compte de la faiblesse de la bourgeoisie. Les grands féodaux, très influents, avaient les mêmes intérêts que les Ottomans et ne se révoltaient que pour défendre leurs privilèges. La bourgeoisie, dans l'espoir de se concilier l'appui indispensable des chefs conservateurs dans la lutte pour l'autonomie, évitait de poser la question de la réforme agraire, alors que celle-ci était posée par les autres mouvements nationaux balkaniques. Le facteur externe est le manque de soutien international. Cet ensemble de faiblesses menaçait le peuple albanais dans son existence même. D'où les multiples appels à l'unité, véhiculés, comme bien d'autres messages politiques de ce temps-là, sous forme de poèmes et de chants.

La crise d'Orient qui commença en 1875 plaça le mouvement national albanais devant des choix difficiles, le Protocole de Londres (mars 1877) ignorant les Albanais. Le 
dilemme était de s'engager contre l'Empire au côté des peuples chrétiens qui déniaient l'existence d'une nationalité albanaise et qui renouvelaient leurs prétentions sur les terres albanaises ou de se ranger sous les bannières du sultan mais d'obtenir de lui en contrepartie la reconnaissance de cette nationalité. À l'initiative d'Abdul Frashëri, se tint à Janina une réunion de notables qui réclama l'autonomie de toute l'aire de peuplement albanais, la réunion en une seule province des quatre vilayets qui partageaient l'aire de peuplement albanais, avec une administration locale et la langue albanaise dans l'administration et l'enseignement. Le mémorandum de Janina demeura cependant sans effet. De plus, durant la guerre russo-turque de 1878, les Serbes envahirent le Kosovo, les Monténégrins la région de Shkodra, les Grecs celle de Saranda, tandis que le traité de San Stefano (mars 1878) englobait dans la Grande Bulgarie Korça, Pogradec, Dibra, Gostivar et Tetova. Face à ce démembrement, Abdul Frashëri convoqua des représentants des trois confessions. Les 80 délégués, venus des quatre vilayets peuplés d'Albanais, Kosovo, Shkodra, Monastir et Janina, décidèrent, selon la vieille tradition albanaise, de former une "ligue nationale", passée dans l'histoire sous le nom de Ligue de Prizren. Elle fut cependant tiraillée entre les tendances conservatrices et nationalistes. Les conservateurs, représentés par l'aristocratie beylicale et le clergé musulman, étaient soucieux surtout de maintenir l'intégrité de l'Empire contre les nationalismes chrétiens, ce qui préserverait à la limite l'intégrité des terres albanaises. Les autres mettaient au premier plan l'union des Albanais des trois confessions pour imposer à la Porte l'autonomie du pays à l'intérieur de l'Empire.

La Ligue de Prizren avait du moins posé la question albanaise devant l'opinion internationale. En effet, aucune des puissances participant au Congrès de Berlin ne s'attendait que leurs décisions concernant les territoires albanais puissent susciter une telle opposition de la part des populations locales organisées par la Ligue de Prizren. C'est ce qui devait les obliger, pendant plus de trois ans, à revenir plus d'une fois sur les décisions qu'elles prenaient. Toutefois la revendication politique moderne de l'autonomie et de l'indépendance nationale ne l'emporte clairement chez les Albanais qu'à partir de 1909.

24 En ce début du XX⿳亠㐅⿵冂⿰㐅㐅乂 totalité des régions albanaises appartenaient à l'Empire ottoman (à quelques détails près : Ulqini, par exemple, était au Monténégro depuis 1880). Le mouvement national albanais, pris entre la répression ottomane et les ambitions territoriales des États balkaniques et sans grand appui extérieur, ne put obtenir l'indépendance que d'à peine la moitié de l'aire de peuplement albanais dans les Balkans. À partir de 1913, une grande partie des Albanais va constituer une minorité nationale au sein de deux Étatsnations agrandis, la Serbie et le Monténégro, puis au sein d'un État plurinational qui tente de fonctionner comme un État-nation, le royaume des Serbes, Croates et Slovènes. D'autres Albanais, aussi nombreux, se sont trouvés en Grèce. ${ }^{11}$ Le reliquat est enfin contenu dans les frontières d'une Albanie sous-dimensionnée.

\section{L'affrontement des nationalismes}

Les circonstances qui ont déterminé, à l'issue des guerres balkaniques, l'établissement d'une frontière coupant en deux, ou plutôt en trois, ce territoire, sont fort complexes car elles mettent en jeu, outre les rapports conflictuels du gouvernement ottoman avec 
ses sujets albanais, les intérêts de quatre États nationaux déjà constitués dans les Balkans et ceux de six grandes puissances européennes.

Les arguments majeurs de cette amputation (au moins en ce qui concerne les régions du nord-est, au sud la situation n'étant pas d'ailleurs différente) restent toujours les prétentions serbes que ces régions appartenaient historiquement aux Serbes. Or l'application de la conception rétrospective serbe se heurtait à une difficulté majeure. Cette région, récupérée, était en majorité peuplée d'Albanais. D'où un investissement intellectuel énorme dans une querelle déjà ancienne du premier occupant, une querelle attisée et exacerbée à tout moment. L'historiographie serbe du XIX ${ }^{\text {ème }}$ siècle s'est efforcé de démontrer que les Albanais sont des tard-venus dans les Balkans. On les supposait, entre autres hypothèses, originaires du Caucase. Les Albanais, au contraire, se sont affirmés descendants des Illyriens. Le Kosovo, la Dardanie antique, relativement épargnée par les invasions slaves des VI-VII ${ }^{\mathrm{èm}}$ siècles qui, descendant des contrées danubiennes, se dirigeaient vers Thessalonique, aurait eu constamment un peuplement majoritairement albanais. Débats et controverses sur les origines illyriennes des Albanais se sont pourtant succédés depuis le XVIII ${ }^{m e}$ siècle parmi les spécialistes dans l'affrontement des thèses thrace ou pélasgienne, voire même caucasienne.

Malgré un hiatus de preuves historiques du VII ${ }^{\text {ème }}$ au XI ${ }^{\text {ème }}$ siècles, ils ont avancé à l'appui de la thèse illyrienne des arguments d'ordre linguistique, essentiellement toponymique, ou de continuité de la culture matérielle. Pour la grande majorité des historiens médiévistes et la plupart des savants linguistes, l'absence de documents historiques n'est pas déterminante. Selon eux, les Illyriens ne disparurent pas entièrement en tant qu'ethnie. Les populations qui échappèrent à la romanisation et à l'extermination de la part des Barbares, ne furent slavisées que dans le Nord et à l'Est de l'Illyrie, alors qu'à l'Ouest elles échappèrent à la slavisation en se repliant dans les montagnes, loin de l'attention des écrivains et des chancelleries de l'époque. Les opinions en matière de définition de cette zone de repli ne sont cependant pas unanimes. Thunmann (1774), Leake (1812), Hahn (1853), Fallmerayer (1861), Sufflay (1913), Stadtmüller (1941) donnent chacun pour leur part des délimitations différentes de la zone. De leur côté, un grand nombre de linguistes, étudiant certains mots rares et un échantillon important de noms de lieux et de personnes légués par le monde illyrien, sont arrivés à la conclusion que la langue albanaise est en rapport de filiation directe avec l'illyrien, plus précisément avec l'un de ses dialectes.

Vers la fin du XVIII ${ }^{\text {ème }}$ siècle apparaît la thèse dite thrace, soutenue aujourd'hui principalement par certains historiens et linguistes roumains ou serbes. Les principaux arguments à l'appui de cette thèse relèvent de la linguistique. Il s'agit de quelques rares mots que l'albanais a hérité du thrace, d'ailleurs tout aussi inconnu que l'illyrien. Il s'agit aussi de quelques autres vocables du dialecte latin du Danube, qui ont pénétré dans l'albanais et d'une série de mots albanais qui, de très bonne heure, se sont introduits dans le roumain. Sur la base de ces éléments, les tenants de cette thèse estiment que les Albanais sont les descendants non des Illyriens mais d'une tribu thrace. Ils pensent que leur première patrie est à rechercher non pas sur les rivages de l'Adriatique, mais dans les profondeurs des Balkans, à proximité des régions où se parlait le latin danubien et au voisinage des lieux où se constitua le peuple roumain. Les Albanais auraient quitté leur premier habitat pour parvenir à leur patrie actuelle au cours des migrations barbares (pour certains antérieurement à la venue des Slaves, 
pour d'autres postérieurement) demeurant ainsi, durant tout le haut Moyen Âge, comme un îlot dans l'océan slave.

On connaît bien aujourd'hui, à partir de sources narratives, la liste des tribus slaves établies au cours du VII ème siècle dans la péninsule balkanique. Leur répartition cartographique indique que durant ce siècle le gros des Slaves s'était fixé, pour ce qui concerne l'Illyrie, dans les régions actuelles de Slovénie, de Croatie, de Serbie, de Bosnie, d'Herzégovine, de Macédoine et dans l'intérieur de la Dalmatie. En dehors de ces pays, des masses importantes s'établirent en Thrace et certaines tribus en Thessalie et dans le Péloponèse. Aucune source historique du VII ${ }^{\text {ème }}$ siècle ne parle d'établissement de tribus slaves dans la Nouvelle Épire, ni dans l'Ancienne Épire ou en Dardanie. Constantin Porphyrogénète mentionne une seule tribu slave, celle des Diocléens, installée selon lui dans la partie septentrionale de la Prévalitanie, approximativement sur les territoires du Monténégro actuel. Des Miracula sancti Demetrii et de la recension arménienne de Ptolémée, il ne ressort pas non plus que des tribus slaves se soient établies dans ces quatre provinces. Ainsi, si l'on tient compte du fait que dans l'Antiquité le sud de l'Ancienne Épire n'était pas habité par les Illyriens, mais par les Hellènes, il apparaît que de tout le vaste pays des Illyriens de l'époque antique, les seules régions qui ne se soumirent pas à la colonisation slave du VII ${ }^{\text {ème }}$ au IX ${ }^{\text {ème }}$ siècles étaient la Nouvelle Épire, la Dardanie, le sud de la Prévalitanie et le nord de l'Ancienne Épire, ce qui correspond à l'aire de peuplement albanais depuis le Moyen Âge jusqu'à l'heure actuelle (Albanie, Kosovo et Macédoine occidentale).

D'autres chercheurs considèrent que l'importance de la toponymie slave dans tous les Balkans, y compris l'aire de peuplement albanais, serait déterminante d'une slavisation de longue date de la région. Néanmoins, la toponymie des premiers siècles du Moyen Âge est inconnue. Dans les documents historiques, les noms de lieux slaves n'apparaissent qu'assez tard, vers le XI ${ }^{\text {ème }}$ siècle. Et souvent ce sont des dénominations imposées par l'administration du royaume bulgare qui, du IX au XI ${ }^{\text {ème }}$ siècles, exerça sa domination sur l'Ancienne Épire et la Nouvelle Épire, ou par celle des États serbes qui englobèrent, pour une plus longue période les provinces de Prévalitanie et de Dardanie. Que ces premiers noms de lieux slaves aient été au XI ${ }^{\text {ème }}$ siècle d'origine récente est attesté par le fait qu'au $\mathrm{X}^{\mathrm{ème}}$ siècle, le basileus Constantin Porphyrogénète appelait encore Byllis, Amantia, Pulcheropolis par leurs anciens noms, alors que l'empereur Basile II, 60 ans plus tard, citait ces mêmes villes sous les noms de Gradetsin, Glavinica, Belegradon (le Berat actuel, en Albanie du Sud). En plus, les noms de lieux slaves que l'on connaît au Xì̀me siècle sont en nombre négligeable, par rapport aux noms de lieux non slaves que les sources du haut Moyen Âge ont transmis pour cette région.

Il convient de tenir compte ensuite d'un autre facteur de la plus grande importance : les matériaux archéologiques concernant la période allant $d u V^{\text {ème }}$ au IX ${ }^{\text {ème }}$ siècles. Une poterie particulière, d'usage domestique, est répandue sur toute l'aire de peuplement albanais et nombre d'objets de parure métallique trouvés dans la moitié nord de l'Albanie s'apparentent à ce qu'on a appelé la culture de Koman (du village de Koman dans la région de Puka à l'est de Shkodra où en 1898 ont été découverts les premiers vestiges). Ils sont de facture essentiellement locale et témoignent d'une continuité incontestable de la tradition illyrienne, en tout cas préslave, encore qu'elle revête parfois des expressions différentes sous l'influence du goût de l'époque et des produits étrangers. À leurs côtés, on trouve aussi des objets métalliques fabriqués par des maitres locaux d'après des modèles romains de la période impériale ou des prototypes 
byzantins, qui attestent que les hommes de Koman ont vécu sans discontinuité dans ces régions tout au long de l'histoire ancienne, médiévale et moderne.

Si les intellectuels serbes ont admis ensuite l'antériorité de la présence albanaise dans les Balkans (Cvijić même admet leur descendance illyrienne ${ }^{12}$ ), ils se sont généralement efforcés d'en minimiser l'importance. L'argument ab antiquo concernant le passé historique du Kosovo n'a pas cessé d'être utilisé pour souligner l'importance de la région pour la nation serbe. Il est toujours et de plus en plus répété que le Kosovo constitue le mythe central de la conscience nationale serbe, comme le "berceau" même de leur État médiéval et de leur Église. La meilleure preuve en est notamment la construction, lors du développement de l'État serbe de la dynastie des Nemanjić, de nombreux édifices religieux orthodoxes. Ainsi, aux XII-XIV ${ }^{\text {ème }}$ siècles, les Albanais n'auraient constitué qu'une population résiduelle, pastorale et montagnarde, presque absente du Kosovo dont la toponymie est principalement slave.

En fait, ceci renvoie plutôt au pouvoir qu'ont détenu les Serbes sur cette région depuis leur arrivée au VI-VII ${ }^{\text {ème }}$ siècles et à leur foi chrétienne. Mais ceci ne signifie nullement que les Albanais étaient absents sur ces territoires avant la domination ottomane. On sait qu'une fois la conquête d'un territoire accomplie, qu'elle soit militaire ou pacifique, politique ou économique, la première chose à faire c'est de construire l'église de $s a$ religion comme une preuve d'apporter la civilisation, ce qui ne témoigne en fait que d'une supériorité de statut. C'est ce que tous les colonisateurs et conquérants ont fait, Christophe Colomb au Nouveau Monde, Constantin à Byzance, Justinien à Ravenne, pour ne citer que les cas les plus parlants. L'Empire médiéval serbe ne saurait être autre chose qu'un empire multiethnique, comparable à celui de Charlemagne, et où les Albanais étaient une importante partie. Ce fait serait toutefois masqué au temps de l'Empire serbe par la conversion de nombreux Albanais catholiques à l'orthodoxie et par leur adoption d'une anthroponymie slavo-orthodoxe. En outre, les sources serbes médiévales n'auraient distingué que les Albanais catholiques, qualifiant les autres de Serbes $^{13}$. Car il est tout à fait remarquable de ne pas mentionner l'existence de nombreux monuments religieux et culturels albanais dans la région. Les églises catholiques du Kosovo, par exemple, sont parmi les édifices religieux les plus anciens dans la région. D'autant plus que les églises et les monastères orthodoxes au Kosovo ont survécu au-delà des cinq siècles d'occupation ottomane notamment grâce à la protection par les Albanais, malgré leur conversion ultérieure à la religion islamique du nouveau conquérant.

Le passé serbe du Kosovo pourrait très bien se comparer aux relations entre l'Empire byzantin, le Patriarcat orthodoxe d'Istanbul et les Grecs d'aujourd'hui, à l'Empire carolingien de Charlemagne, sa capitale située en Aix-la-Chapelle et les Français d'aujourd'hui, ou encore à l'Espagne occupée par les Arabes. Ni les Grecs, ni les Français, ni les Arabes ne peuvent aujourd'hui avoir ouvertement des revendications de "droits historiques" sans devenir ridicules.

À part le fait surprenant que la communauté internationale soit préoccupée, au jour d'aujourd'hui même, par l'héritage historique des nations, ce qui parait encore plus intrigant est qu'on ne donne pas le même poids au fait que le Kosovo est aussi le "berceau" de l'État albanais à la fois médiéval et moderne. L'État médiéval albanais de la dynastie des Balsha, surnommés les "maîtres de l'Albanie", s'étendait dans la région depuis la moitié du $13^{e}$ siècle jusqu'à la fin du XIV ème siècle. La Bataille de Kosovo qui a nourri le mythe serbe de la victimisation ne fut pas la bataille des Serbes pour "sauver 
la Chrétienté", comme on l'entend toujours. La Bataille de Kosovo fut menée en 1389 par une coalition régionale où les Albanais étaient largement partie prenante. Un des triumvirs qui a conduit l'Armée de la coalition, avec le Prince serbe Lazar Hrebeljanović et le Roi Tvrtko de Bosnie, était le "Maître" des Albanais Georges II Balsha, alors que Théodore II Muzaka, le Prince de Berat en Albanie du Sud, perdit plus de 4000 hommes dans le champ de la bataille.

$\mathrm{Au}$ siècle suivant, la formidable épopée de la résistance de Skanderbeg prouve bien, s'il en était besoin, que les Albanais ne se sont point résignés après la bataille de Kosovo. Une des pages les plus illustres de l'histoire des Albanais fut écrite notamment, sous la conduite de Skanderbeg devenu leur héros national, pour empêcher le débordement des Ottomans vers l'Occident. Issu d'une maison albanaise de moyenne noblesse, Georges Kastriote dit Skanderbeg est né vers 1403 et élevé en otage chez les Ottomans où il a reçu le surnom d'Iskander bey, "prince Alexandre", imaginé par la suite comme un signe de reconnaissance de son ascendance illyrienne, faisant allusion à Alexandre le Grand. Il a déserté en 1443 lors de la défaite ottomane devant les Hongrois à Nis (Naissus). À la tête d'une petite troupe de cavaliers, 300 dit la tradition orale, il s'est emparé de Kruja, non loin de Durrës et de Tirana. Le lendemain, il hissait sur la citadelle le drapeau aux armes de sa famille: double symbole chargé d'avenir. Le drapeau de Skanderbeg allait devenir l'emblème de l'Albanie indépendante et la date même de la proclamation de l'indépendance en 1912, le 28 novembre, qui restera celle de la fête nationale albanaise, fut imaginée comme une réplique à ce jour ancien. En 1444, Skanderbeg fut proclamé prince des Albanais lors de la Ligue de Lezha (Alessio), dans la ville côtière contrôlée à l'époque par les Vénitiens. Il a combattu depuis contre les Turcs ottomans qu'il a pu arrêter aux portes de l'Europe durant plus d'un quart de siècle, à l'apogée même de leurs conquêtes européennes. Pratiquement seul, c'est grâce à son adresse personnelle qu'il a réussi à arrêter plusieurs offensives, conduites non seulement par les meilleurs hommes de guerre ottomans tels Evrenos Bey ou Ballaban Pacha, qui a péri au combat, mais aussi par les deux plus puissants Sultans des Turcs ottomans eux-mêmes : Murâd II qui est mort en 1451 après un long siège échoué devant les murs de Kruja, dont le héros albanais avait fait sa capitale, et Mehmet II Al Fâtih, le "Conquérant" de Byzance et des Balkans, qui a mis trois fois le siège devant Kruja sans pouvoir lui venir à bout. En janvier 1468, terrassé par une attaque de fièvre, Skanderbeg mourait invaincu à Lezha. Après sa mort, le relais a été pris par l'un de ses principaux généraux, Lekë Dukagjini, dernier descendant d'une grande maison de la noblesse albanaise, reconnu aussi par la tradition orale comme le premier législateur albanais. Il a fallu encore plus d'une dizaine d'autres longues années pour que les Ottomans arrivent à s'emparer des dernières forteresses et annexer le pays albanais.

Quant à l'importance de la région du Kosovo pour l'unité albanaise, il est notable que dans les temps modernes la première Ligue nationale des Albanais, après celle de Skanderbeg, fut rassemblée en 1878 dans le ville de Prizren au Kosovo, suivi par une deuxième Ligue nationale en 1899 dans la ville de Peja également au Kosovo.

Un autre argument est que les Albanais ne seraient venus au Kosovo, pour l'essentiel, que tardivement, après la conquête ottomane, le pouvoir ottoman favorisant cette expansion suite à leur conversion majoritaire à l'islam. C'est ainsi, en particulier, qu'ils auraient comblé le vide laissé par la grande migration des Serbes de Kosovo vers le bassin pannonien, en 1690, à la suite de la première guerre austro-turque. Leur expansion se serait poursuivie aux XVIII-XIX ${ }^{\text {ème }}$ siècles, s'étendant toujours plus loin 
vers le nord-est. Les historiens albanais, par contre, soutiennent que l'idée d'une migration massive des Serbes s'est imposée au XIX ${ }^{\text {ème }}$ siècle sans examen critique. On aurait en fait mal interprété les documents de l'époque, prenant ainsi pour une élimination totale de la population orthodoxe ce qui n'était que la destruction de son encadrement ecclésiastique ${ }^{14}$, position déjà soutenue par Jovan Tomitch ${ }^{15}$. En outre, la promptitude de la reconquête ottomane, lors de la retraite des Impériaux, aurait rendu impossible la fuite de la population. Enfin, tout cela se passait au cœur de l'hiver, ce qui rend invraisemblable un exode massif.

L'émigration chrétienne aurait donc été modeste, et d'ailleurs en partie albanaise, l'évêque catholique albanais de Scopia, Pjeter Bogdani, ayant appelé à la révolte contre les Turcs en même temps que le patriarche orthodoxe serbe Arsenije Carnojević. Toutefois, alors que Tomitch estime que les Serbes, privés de leur encadrement ecclésiastique, ont été ensuite islamisés et albanisés, Juka, déclarant ne pas voir pourquoi ils auraient été albanisés, conclut qu'ils étaient très peu nombreux dans ces régions avant 1690 et que, de ce fait, les Albanais y étaient déjà largement majoritaires. D'ailleurs, aucun scénario démographique n'a pu résister au fait simple que les montagnes d'Albanie du nord, d'où les historiens serbes les font descendre, n'auraient pu fournir assez de monde pour repeupler un Kosovo déserté ${ }^{16}$. Enfin, le Kosovo n'aurait pas été islamisé en masse au cours du siècle suivant, mais l'aurait déjà été aux deux tiers dès le XVI ${ }^{\text {ème }}$ siècle. Rizaj met également en doute l'existence de la grande migration serbe ${ }^{17}$, de même que Pulaha qui cite des sources autrichiennes de cette époque nommant le Kosovo "Albanie" et faisant état d'insurgés albanais ${ }^{18}$.

\section{Les relations interethniques}

Même si des incertitudes demeurent, dues à la faiblesse de raisonnements reposant en partie sur des supputations et sur une utilisation sélective de sources d'ailleurs insuffisantes pour permettre une connaissance précise des mouvements démographiques à cette époque, il paraît aujourd'hui acquis, à la suite des travaux des historiens albanais sur l'onomastique des cadastres ottomans, que les Albanais constituaient une partie importante de la population du Kosovo. Acquis, du moins, chez les historiens spécialistes de la question, car la version serbe traditionnelle est presque universellement répandue à l'étranger, où elle se perpétue comme une idée reçue et vient d'être réactivée par les nombreuses publications réalisées récemment. Cependant, en ce qui concerne l'aire de peuplement albanais en ex-Yougoslavie, actuellement il s'agit d'une population concentrée, contrairement à d'autres comme les Tsiganes et les Valaques. Elle est majoritaire dans ce territoire, malgré le maillage politico-administratif qui coïncide mal avec le territoire ethnique. La Province autonome de Kosovo, par exemple, n'est qu'une approximation institutionnelle de l'ensemble précédent. On est donc souvent réduit à considérer le Kosovo comme un succédané de l'aire de peuplement albanais, faute de disposer de données suffisantes pour le reste de cette dernière.

41 En tout cas, il est probable que des phénomènes d'assimilation ont existé dans les deux sens, entre Serbes et Albanais, selon les lieux et les circonstances. Au demeurant, l'assimilation n'est pas seulement affaire de violence, institutionnelle ou non. Elle implique aussi une adhésion collective implicite aux valeurs véhiculées par la langue dominante. En d'autres termes, il faut être dans une certaine mesure consentant pour 
être assimilé. Mais, occupés à exalter les différences entre leurs peuples, les intellectuels des deux bords ne se sont jamais intéressés à ce qu'ils ont en commun.

La question nationale se situe au point d'intersection entre la politique, la technologie et la transformation des sociétés. Les nations existent non seulement en tant que fonctions d'un type particulier d'État territorial ou de l'aspiration à en établir un, mais aussi dans le contexte d'une étape particulière du développement technologique, économique et culturel. Aujourd'hui, la plupart de ceux qui se penchent sur la question s'accorderont pour dire que les langues nationales unifiées, parlées ou écrites, ne peuvent apparaître en tant que telles avant l'imprimerie, l'accès d'une grande majorité des gens à la lecture et donc l'instruction de masse. Il faudra donc analyser les nations et les phénomènes qui leur sont associés en termes politiques, techniques, administratifs, économiques, culturels, et en tenant compte de tout ce qu'exigent les conditions particulières.

En tout cas, à l'encontre de l'histoire culturelle albanaise, l'énorme retard de l'alphabétisation ne doit pas inciter à considérer les Albanais comme un peuple inculte, comme si la culture orale n'était pas une culture, et encore moins à gommer les percées culturelles régionales, notamment dans le sud. Il est important d'envisager le décalage croissant entre une Europe de plus en plus systématiquement vouée à l'enseignement de masse et au règne de l'écrit et une partie de la population dont la culture continuait à être produite et transmise par voie orale : population plus ou moins enclavée selon les régions, privée des codes d'accès à une vie culturelle plus large, ce qui accentuait en même temps les différences culturelles au sein même de la société albanaise, notamment entre le nord et le sud. L'ignorance de la langue officielle, les différences de religion, l'analphabétisme, les conflits agraires et les vexations des autorités creusent ainsi un fossé entre les masses albanaises d'un côté et les représentants de l'État de l'autre, hier envers la domination ottomane comme aujourd'hui envers l'administration yougoslave (en ce qui concerne au moins les Albanais au Kosovo et en ex-Yougoslavie).

Peu familiers de l'administration et des tribunaux, dont ils craignent l'arbitraire, surtout dans les régions du Kosovo et de l'Albanie du nord, les Albanais continuaient à régler fréquemment leurs différends entre eux en se fondant sur leurs droits coutumiers. Les codes de conduite auxquels se sont référés les Albanais sont au nombre de trois : ligji i shtetit, la loi de l'État qui a pris actuellement le pas sur les deux autres, sheriati, la loi islamique pour la majorité musulmane ou ligji $i$ kishes, la loi canonique respectée surtout par les catholiques dans les régions du nord, mais qui à vrai dire n'ont jamais eu l'influence décisive, et ligji i fshatit, la loi du village, c'est-à-dire le droit coutumier local, naguère prépondérant ${ }^{19}$. Le renversement opéré dans les rapports entre les trois codes est évident, que ce soit en Albanie ou en Yougoslavie. Toutefois, les évolutions récentes dans les deux milieux ont fait remarquer que les intéressés se réfèrent de plus en plus au code coutumier, non seulement parce qu'ils le considèrent comme le seul spécifiquement albanais, mais aussi et surtout parce qu'il ne prévoit pas d'intervention de l'État. Par les temps de crises que traverse la société albanaise actuelle, plus particulièrement en Albanie, on revient de plus en plus aux anciennes valeurs normatives, qui sont constamment mobilisées à des fins productives pour pallier à la rupture des liens sociaux.

Il n'est pas question d'approfondir ici l'étude de la question complexe de l'origine des codes coutumiers albanais ni les questions historiques connexes d'une "société sans 
État" depuis l'origine. Beaucoup d'observateurs étrangers, et même d'Albanais, atteints $\mathrm{du}$ "virus de l'essence", selon l'expression de Roland Barthes, ont succombé à la tentation de répondre directement par l'affirmative, du fait de leur croyance dans l'irréductible originalité albanaise, ou bien dans l'anarchie et l'inaptitude de ce peuple à constituer un État organisé. Cette question est liée à celle, également controversée, du tribalisme des montagnes du nord.

Ce qu'il faut d'emblée souligner, c'est que l'idée de nation s'enracine dans les différences. La cohésion n'est acquise qu'au prix d'une opposition flagrante ou virtuelle à tout ce qui est étranger. Ainsi, l'une des dernières nationalités apparues en Europe, la nation albanaise est restée longtemps marquée par des traits perçus comme archaïques, tels qu'une organisation partiellement tribale, la persistance des communautés familiales et l'usage de la vengeance: caractères que leurs voisins immédiats et la majorité des voyageurs occidentaux, du milieu du XIX ${ }^{\text {ème }}$ siècle aux années 1930, ont "naturalisés" pour en faire l'essence même de ce peuple. Interprétation trop simple, dans la mesure où il ne s'agit pas seulement d'une culture traditionnelle, mais aussi d'une culture refoulée, marginalisée, exclue des voies ordinaires de développement. Face à l'emprise croissante d'États successifs qui les rejetaient et dans lesquels ils ne pouvaient se reconnaitre, les Albanais ne pouvaient que s'affirmer en mettant l'accent sur les aspects les plus traditionnels de leur culture. Ils s'attribuaient l'exclusivité ethnique de ce qui pouvait n'être que le signe d'un décalage, l'effet d'une entrave à un besoin refoulé d'affirmation, vers l'extérieur, des formes idéologiques, religieuses ou nationales, de leurs valeurs culturelles. Souvent perçus de façon défavorable par leurs voisins immédiats, ils étaient à leur tour tentés de cultiver leur particularisme, de se poser en s'opposant. Ils ont ainsi résisté à l'assimilation par une autre stratégie de déplacement et de subversion, par la conservation et cette espèce d'“hibernation dans l'histoire", selon l'expression d'Angelo Di Sparti, en projetant à l'extérieur l'idéologie de leur identité collective, ethnique et nationale.

\section{NOTES}

1. Grousset (René), L'Empire du Levant. Histoire de la question d'Orient, Paris: Payot, 1949, p. 7.

2. Kitsikis (Dimitri), L'Empire ottoman, Paris: PUF, 1985.

3. Arensberg (C.M.), "The Old World Peoples. The Place of European Cultures in World Ethnography ", Anthropological Quarterly, 36 (3), 1963.

4. Doja (Albert), «À propos de la diversité locale des traditions culturelles albanaises », La Ricerca Folklorica, 38, 1998.

5. Ancel (Jacques), Peuples et nations des Balkans: Géographie politique, Paris : Éditions du Comité des Travaux Historiques et Scientifiques, 1992 [éd. originale : 1930] ; Jelavich (Charles), Jelavich (Barbara), The Establishment of the Balkan National States (1804-1920), Seattle: University of Washington Press, 1977.

6. Roux (Michel), Les Albanais en Yougoslavie. Minorité nationale, territoire et développement, Paris : Éditions de la Maison des Sciences de l'Homme, 1992. 
7. Mauss (Marcel), CEuvres, vol. 3, Paris : Éditions de Minuit, 1969, p. 586.

8. Roux (Michel), op.cit., pp. 168-169.

9. Doja (Albert), «L'idée de nation : du postulat de Marcel Mauss à la question actuelle des identités nationales ", Revue de l'Institut de Sociologie, (1-4), 1996.

10. Haxhihasani (Qemal), Chansonnier épique albanais, Tirana: Académies des Sciences, 1983 ; Fetiu (Sadri), Këngë popullore të Rilindjes Kombëtare të Lidhjes së Prizrenit, Prishtina: Institut Albanologique, 1978.

11. Le découpage des territoires albanais, notamment le rattachement du Kosovo et de la Macédoine occidentale à la Serbie, comme le rattachement de la région de Çamëria à la Grèce, fut effectué sur décision de la Conférence des Ambassadeurs des six Grandes Puissances européennes tenue le 29 juillet 1913 à Londres au lendemain des Guerres balkaniques.

12. Cvijic (Jovan), La Péninsule balkanique. Géographie humaine, Paris: Armand Colin, 1918.

13. Pulaha (Selami), L'autochtonéité des Albanais en Kosova et le prétendu exode des Serbes à la fin du $17^{e}$ siècle, Tirana : 8-Nentori, 1985.

14. Juka (Safete), Kosova. The Albanians of Yugoslavia in Light of Historical Documents, New York: Waldon Press, 1984.

15. Tomitch (Jovan), Les Albanais en Vieille-Serbie et dans le Sandjak de Novi-Bazar, Paris : Hachette, 1913

16. Roux (Michel), op.cit.

17. Rizaj (Selman), «Mbi te ashtuquajturen dyndje e madhe serbe nga Kosova me ne krye patrikun Arsenije Carnojeviq (1690) » (À propos de la soi-disant grande migration des Serbes de Kosovo sous le patriarche Arsène Carnojevic, 1690), Gjurmime Albanologjike, Série historique, 12, 1983.

18. Pulaha (Selami), op.cit., pp. 74-81.

19. Il existait plusieurs normes juridiques traditionnelles dans l'aire de peuplement albanais. Le droit coutumier le plus connu est le Kanuni i Lekë Dukagjinit, que la tradition fait remonter au prince Lekë Dukagjini, compagnon de Skanderbeg, sans qu'on puisse confirmer la validité de cette relation. La plupart des travaux ethnographiques dénomment par ce terme les normes juridiques coutumières observées dans la région de Dukagjini en Albanie du Nord et sur le Plateau de Dukagjini jusqu'au Kosovo, c'est-à-dire sur le territoire des anciens domaines de la principauté des Dukagjini. Elles ont été recueillies dans les régions du Nord et codifiées par le prêtre catholique Shtjefen K. Gjeçov, de Janjevo (Kosovo), à la fin du XIX ${ }^{\text {ème }}$ siècle et au début du $X^{\text {ème }}$ siècle. Son recueil posthume fut publié par les soins des Fransiscains de Shkodra en 1933. Une deuxième édition, augmentée de manuscrits inédits, ne sera publiée par les soins de l'Académie des Sciences d'Albanie qu'en 1989, entretemps il fut publié en italien, en allemand et récemment en anglais (Gjeçov (Shtjefën K.), Kanuni i Lekë Dukagjinit (Le Code de Leka Dukagjini) [Publié et annoté par K. Nova], Tirana: Académie des Sciences, 1989 (Patrimoine Culturel des Albanais: Droit Coutumier, 1) [1933]). Dans la tradition albanaise, les droits de la coutume s'étendaient pourtant sur un domaine plus large, notamment dans ces régions où ont été repérés les derniers vestiges de l'organisation lignagère de type segmentaire. En Albanie centrale, sur la rive droite de Shkumbini, dans la région de Ghèghenie proprement dite, où s'étendaient les anciens domaines de la principauté de Georges Kastriote, dit Skanderbeg, les travaux ethnographiques ont retenu des normes semblables sous le nom de Kanuni $i$ Arbërisë "Droit d'Albanie" ou Kanuni i Skënderbeut "Droit de Skanderbeg". Dans d'autres régions, notamment dans la région des montagnes d'Albanie du nord depuis la montagne de Shkodra jusqu'à la montagne de Gjakova et la plaine de Kosovo c'est le terme de Kanuni i Maleve "Droit des Montagnes", qui a été retenu. Il est cependant fort probable que ces dénominations locales soient des variantes des mêmes normes juridiques coutumières, observées depuis la vallée de Shkumbini jusqu'au Kosovo et probablement dans le sud-ouest de l'Albanie, dans les régions de Vlora, Kurveleshi, Himara, Tepelena, généralement connues sous le nom de Labëria, jusqu'aux 
montagnes des Souliotes. Dans ces régions du sud-ouest, c'est encore un autre terme, Kanuni $i$ Labërisë "Droit de Labëria", qui a été retenu par les travaux ethnographiques.

\section{AUTEUR}

ALBERT DOJA

Docteur en anthropologie sociale (ÉHÉSS Paris). 